\title{
Quantification of Residual Stress from Photonic Signatures of Fused Silica
}

\author{
K. Elliott Cramer ${ }^{\mathrm{a}}$, Maurice Hayward ${ }^{\mathrm{b}}$ and William T. Yost ${ }^{\mathrm{a}}$ \\ ${ }^{a}$ NASA Langley Research Center, Hampton, VA 23681 \\ ${ }^{b}$ College of William \& Mary, Williamsburg, VA 23185
}

\begin{abstract}
A commercially available grey-field polariscope (GFP) instrument for photoelastic examination is used to assess impact damage inflicted upon the outer-most pane of Space Shuttle windows made from fused silica. A method and apparatus for calibration of the stress-optic coefficient using four-point bending is discussed. The results are validated on known material (acrylic) and are found to agree with literature values to within $6 \%$. The calibration procedure is then applied to fused-silica specimens and the stress-optic coefficient is determined to be $2.43 \pm 0.54 \times 10^{-12}$ $\mathrm{Pa}^{-1}$. Fused silica specimens containing impacts artificially made at NASA's Hypervelocity Impact Technology Facility (HIT-F), to simulate damage typical during space flight, are examined. The damage sites are cored from fused silica window carcasses and examined with the GFP. The calibrated GFP measurements of residual stress patterns surrounding the damage sites are presented.
\end{abstract}

Keywords: Glass, fused silica, photoelasticity, residual stress

PACS: $78.20 . \mathrm{hb}$

\section{INTRODUCTION}

During processing and flight, windows on space vehicles may be subjected to harsh and damaging conditions capable of impairing their mechanical performance. The outer windowpanes on spacecraft encounter high velocity impacts (HVI) from debris and micrometeoroids occurring from vehicle launch to landing. During vehicle re-entry these windowpanes are subjected to high temperatures. While in storage and during preparation for launch, windows sometimes encounter accidental collision events, such as a dropped tool, or a tethered device striking its surface.

Fused silica, which is the primary material of choice for crewed spacecraft windows for the manned spacecraft programs at $\mathrm{NASA}^{1}$, has significant advantages over other transparent materials. Space Shuttle window samples were used for the results presented in this paper, but the results are expected to also apply to future space vehicles where fused silica is a likely choice for windows. These include thermal shock tolerance and superior optical performance. Along with these advantages, however, are properties that make engineering with this material very difficult. Fused silica, like other silica-based glasses, is a brittle material, whose strength can be degraded considerably by sharp object impacts $[1,2]$. Other characteristics of engineering significance include a large scatter in material and fracture properties. In particular, fracture strength exhibits a strong dependence on both surface quality (e.g. presence of micro-cracks and other flaws) and the inevitable internal flaws that are distributed throughout the volume.

The aggressive impact and thermal conditions experienced from launch to landing and ground-based events that degrade mechanical performance can affect vehicle safety. As a consequence of the temperature ranges that are encountered, the window mounts must place the glass panes under minimal mechanical stresses (yet, sufficient to seal the glass to the vehicle). When damaged by impacts and placed under tensile stresses, these panes can suddenly fail. All accumulated damage sites, whether from the active (launch to landing) period or from the storage and maintenance period, must be identified, measured, evaluated, and continually monitored for their effects on the pane's structural performance. Since the service life of a pane is shortened by damage causing events, engineers must ensure that the glass surfaces are carefully inspected prior to every active cycle for safety assurance.

During the duration of the Space Shuttle Program, a vehicle was never lost due to a window failure. This safety record was due in large part to a carefully designed plan and execution of window maintenance procedures. During each mission, thousands of new damage sites are added to each pane. A crew dedicated to window maintenance conducted thorough post flight examinations of the Space Shuttle windows. The examination procedure used various and sometimes specially designed optical instruments. During inspection the emphasis was placed on accurate

${ }^{1}$ In the past, these panes have been manufactured from Corning 7940/7989 Fused Silica glass. 
measurements of the damage surface features (a daunting task requiring human judgment and estimation due to typical damage site irregularity). Each damage site was characterized via measurements of mold impressions (damage depth, damage area) and was recorded on maps of damage site locations. The molds were measured with an optical comparator to determine damage size (cross-section and depth) at each impact site. Depth features as small as $15 \mu \mathrm{m}$ were recorded and mapped for further examination and analysis.

Stress analysis incorporates flaw depths into a static fatigue model to calculate a conservative estimate of the residual strength [4]. For the Shuttle, assessments of flight viability were made in light of the residual strength predictions and the expected pressure loads encountered during launch, orbit, and reentry (glass loses strength when subjected to static stresses and pressure gradients [5]). On the basis of this analysis, decisions on window replacement were made.

As the Shuttle program progressed, ongoing studies of the effects of hypervelocity impacts were performed for improvement of the analytical approaches [6]. Fused silica specimens (retired Space Shuttle windows) were subjected to hypervelocity impacts at the NASA Johnson Space Center's Hypervelocity Impact Testing Facility (HIT-F) located at the White Sands Test Facility in Las Cruces, New Mexico. Generally, NASA defines hypervelocity impacts as impacts involving velocities greater than $5 \mathrm{~km} / \mathrm{s}$. Typically these impacts were made with a two-stage light-gas gun launcher. The impacts were made under various conditions, including the firings of impactors of various materials and at a variety of angles.

During the operations outlined in Reference 3, damage to shuttle windows other than from hypervelocity impacts have also been noted. Specifically, low velocity impacts can cause bruises, a class of impacts that produce underlying damage, but do not create surface disruptions with the same characteristics of the damage accumulation associated with higher velocity impacts. Examples of the sources for this damage type include the dropping of an object or tool onto the window and a tool swinging from a tether that collides with the windowpane during vehicle servicing. Another damage source is the dragging of a dull object across the glass surface. This type of damage falls under a specific category, "chatter checks", and is characterized by a linear, somewhat periodic sequence of small bruises of similar diameter caused by the friction contact between the glass and a dull object.

The purpose of this work is to investigate and report on an alternative to the previously used method of combining mold impressions with static stress models. The investigated technique includes a more reliable and more accurate inspection method, with improved reliability for location, along with a predictive assessment of loss of fracture strength caused by windowpane damage in aerospace vehicles. This new technique uses a Grey Field Polariscope (GFP) to measure stress birefringence [7] (the photoelastic response to stress fields in glass). When the glass is damaged, the speeds of light waves passing through the damage regions are dependent on the local stresses associated with the damage and are related to local stress alignment with the light's polarization states. Utilizing GFP measurements one easily locates and characterizes damage regions by noting locations and variations of light speed with polarization direction. Moreover, the photoelastic region surrounding the damage site extends over much larger distances than the damage site itself, thus making detection easier. Once located, the photoelastic response determines the residual stress field around the damage site.

In this investigation we test the ability of the GFP-based measurements to predict fracture strength on a series of specimens with damage sites that are cored from Shuttle window material. Measurements were taken with a GFP inspection system at and near regions of damage corresponding to two of the four different damage classifications discussed above (HVI and Bruises). We relate measurements of residual stress measured with the GFP around damage sites to the measured fracture strength [8] of the specimens. We develop and validate a method of calibrating the GFP instrument using four-point bending to determine the stress-optic coefficient (K). The stressoptic coefficient connects optical retardation to stress (including residual stress) in a transparent specimen experiencing stress birefringence.

\section{PRINCIPLE OF GREY-FIELD PHOTOELASTICITY}

To measure the effect of residual stress fields left in the glass by the impact events, we use a process that is governed by the principle that regions surrounding damage sites in glass become stress birefrigent ${ }^{2}$. The degree of birefrigence is directly related to the magnitude and extent of the plastic stress field and the elastic stress field that extend beyond the immediate damage site.

The visible light GFP was originally developed by Stress Photonics, Inc. for NASA in order to provide highresolution full-field subfringe photoelastic stress analysis in materials transparent to visible light. In this application

${ }^{2}$ Birefringence is the optical property of a material having a refractive index that depends on the polarization and propagation direction of light. Stress birefringence occurs in isotropic solids that exhibit birefringence under mechanical stress. 
of photoelasticity, the stress field of a test article is evaluated through the use of polarized light. The GFP performs this evaluation by combining a circularly polarized light source with a linearly polarized filter. As the filter is rotated, data is acquired with a video camera that uses synchronous (synchronized with the rotation of the filter) demodulation.

Figure 1 shows a schematic representation of the GFP method using photoelasticity to inspect a specimen that exhibits birefringence when strained. Circularly polarized light is represented by two orthogonal linear polarized light vectors that are out of phase by $\pi / 2$ radians.

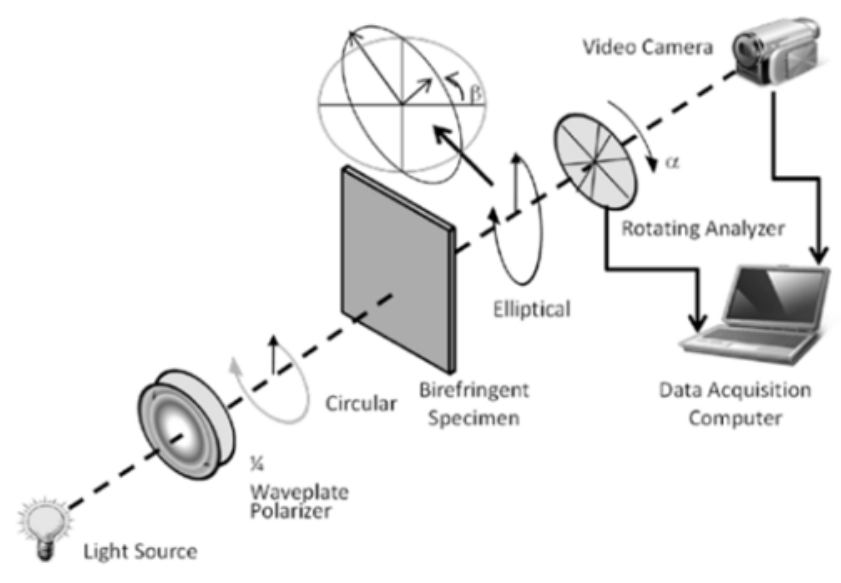

FIGURE 1. Schematic representation of the grey-field polariscope

It has been shown by Horn [9] and Yost [10] that for the GFP configuration described above, the intensity of the optical phase retardation $(\mathrm{R})$ is given by:

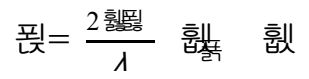

where $\boldsymbol{K}$ is the stress-optic coefficient, $\boldsymbol{b}$ is the thickness of the sample parallel to the optical axis of the system, $\sigma_{x}$ is the first principal stress or stress in the optic x-direction and $\sigma_{y}$ is the second principal stress or stress in the optic ydirection and $\boldsymbol{\lambda}$ is the wavelength of the light used in the measurement.

The data acquisition and initial analysis was obtained with the commercially developed GFP 2400 unit, currently marketed by Stress Photonics [11, 12]. This laboratory instrument is a transmission system that has a spatial resolution determined by the diffraction limits of the lens system and has an optical retardation resolution of less than $0.5 \mathrm{~nm}$.

\section{MEASUREMENT OF THE STRESS-OPTIC COEFFICIENT}

From Equation (1) it can be seen that finding the stress-optic coefficient relates the residual stress within a transparent object with photoelastic retardation by using the GFP. Since no literature values exist for the stress-optic coefficient of the specific fused silica used in this study, it is necessary to determine $\boldsymbol{K}$ experimentally. In order to validate this procedure, measurements were also made on an acrylic (Polymethylmethacrylate or PMMA) specimen and the results compared with the $\boldsymbol{K}$ value in literature. The experimentally determined $\boldsymbol{K}$ for the acrylic specimen was found to agree within one standard deviation of the literature value of the stress-optic coefficient, $4.0 \times 10^{-12} \mathrm{~Pa}^{-}$ ${ }^{1}$ [13], thus validating the adopted methodology.

An apparatus was developed so that beam specimens of interest could be loaded in four-point bending while maintaining a clear optical path for the photoelastic stress measurements. Four-point bending was selected since this allows a one dimensional solution to the stress equation. The general equation for the stress in a beam is:

$$
\text { 휪 }=- \text { 恶 }
$$


where $\boldsymbol{M}$ is the bending moment of the beam, and the $\boldsymbol{y}$ direction is parallel to the applied force (see figure 2). The bending moment is independent of the material used, but depends solely on the laboratory setup of the four-point bending mechanism. I is the moment of inertia and this depends exclusively on the geometry of the beam. The bending moment for four-point bending and moment of inertia for a slender beam are:

$$
\begin{gathered}
\text { 포 }=\frac{\text { 팽 }}{4}[\text { 퍃 폴 }, \\
\text { 팩 } \frac{\text { 풀 }}{12},
\end{gathered}
$$

where $\boldsymbol{F}$ is the force applied to the specimen, $\boldsymbol{a}$ is the distance between the load points and $\boldsymbol{L}$ is the distance between the fixed points in the four-point bending device (Figure 2). Figure 2 shows an outline of the apparatus (with specimen) used for this work, and has the following dimensions: $\boldsymbol{a}=4.17 \mathrm{~cm}$ and $\boldsymbol{L}=6.30 \mathrm{~cm}$. In Equation (3), $\boldsymbol{b}$ is the thickness of the specimen and $\boldsymbol{h}$ is the height, parallel to the direction of force and perpendicular to the optical measurement axis. A beam specimen of acrylic is used for determining $K$ and has a thickness $(\boldsymbol{b})$ of $0.45 \mathrm{~cm}$ and a height $(\boldsymbol{h})$ of $1.45 \mathrm{~cm}$. Four beam specimens of fused silica are used with identical dimensions each will have having a thickness $(\boldsymbol{b})$ of $1.43 \mathrm{~cm}$. and a height $(\boldsymbol{h})$ of $1.28 \mathrm{~cm}$. Substituting Equations (3) and (4) into Equation (2) and solving for $\boldsymbol{K}$ yields:

where,

$$
\text { 퍂= 푠 } h
$$

$$
\text { 푠- } \frac{1}{2 \text { 휠 }} \text {. }
$$

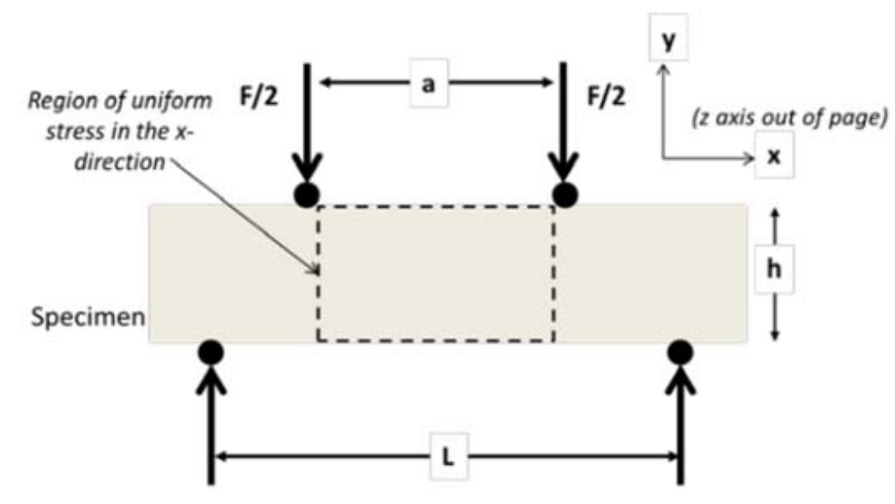

FIGURE 2. An illustration of the four-point bending mechanism designed for this experiment. Where $\boldsymbol{F}$ is the applied force and the optical axis for stress measurements is perpendicular to this drawing, out of the page (z-axis).

Each beam specimen (one acrylic and four fused silica specimens) was placed under four-point bending and data were acquired using the GFP instrument at loads of 8.9, 17.8, 26.7, 35.6 and $44.5 \mathrm{~N}(2,4,6,8$, and $10 \mathrm{lbs})$. A load cell was used to measure the force applied during the measurements.

Figure 3 shows a typical optical retardation image for a specimen under load in the four point bending apparatus. To determine the stress-optic coefficient, a retardation image at each load is recorded and for each row (x) of pixels in the uniform stress region an average stress value is determined and plotted against vertical (y) position. Figure 4 is an example plot for the five different loading cases of the acrylic specimen. The slope of the each of the curves in Figure 4 corresponds to the parameter $\boldsymbol{S}$ in Equations 6 and 7. Using this slope, the load, and the physical parameters of the four point bending apparatus; the photoelastic coefficient was determined to be $3.77 \pm 0.41 \times 10^{-12}$ $\mathrm{Pa}^{-1}$ for acrylic and $2.43 \pm 0.54 \times 10^{-12} \mathrm{~Pa}^{-1}$ for fused silica. For acrylic, the experimentally determined value of the photoelastic constant agrees with the literature value to within $6 \%$. Table 1 provides a complete list of all of the measured values for the photoelastic coefficient for all four fused silica specimens. 


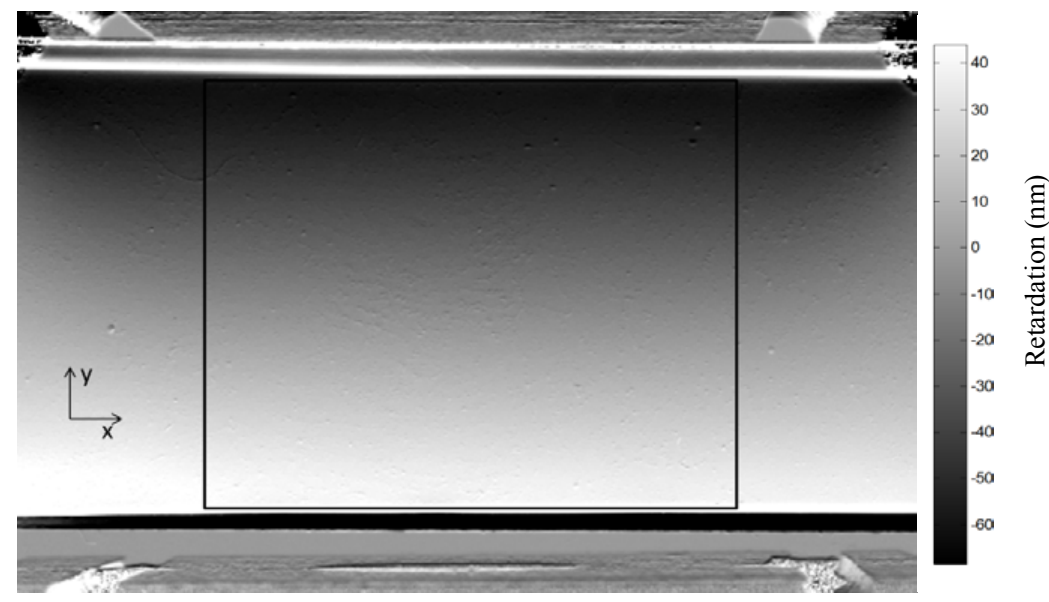

FIGURE 3. Representative optical retardation image $(\mathrm{nm})$ of an acrylic specimen under four point bending. The rectangular region defined by the black lines indicates the area of uniform stress used for averaging.

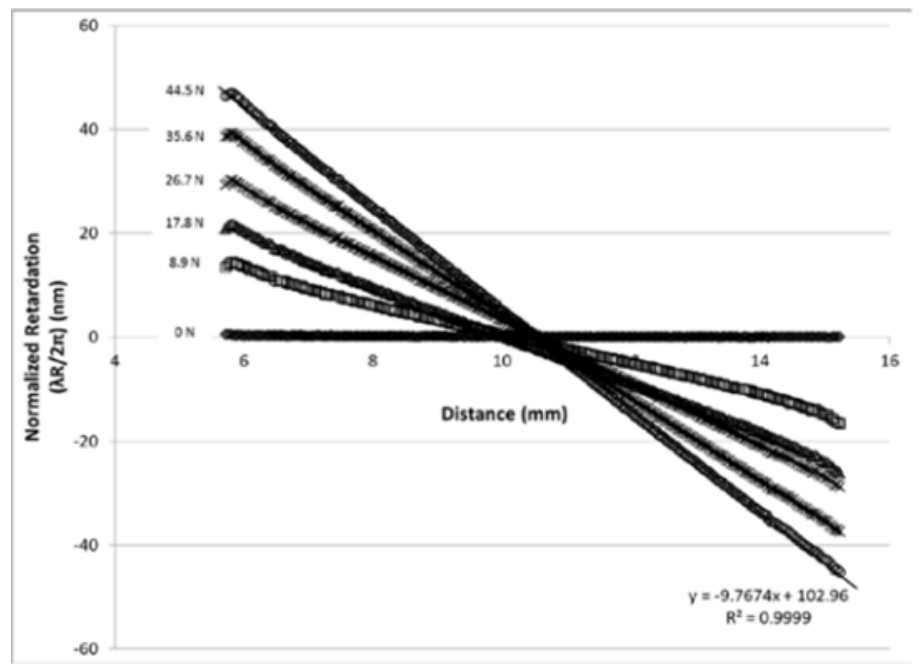

FIGURE 4. Representative retardation vs. position (y) plot for the acrylic specimen at $0,8.9,17.8,26.7,35.6$ and $44.5 \mathrm{~N}$. loads. A typical least squares curve fit is shown for the $44.5 \mathrm{~N}$ load case.

\section{APPLICATION TO DAMAGED FUSED SILICA SPECIMENS}

Specimens were prepared from windows taken from Shuttle vehicles. The two general classes of damage in this study are (1) natural hyper velocity impacts (HVI) encountered during shuttle flight, and (2) bruises created from impacts from low-velocity masses. Other classes of damage commonly observed on vehicle windows, such as chatter checks or a linear series of bruises inflicted by dull and slow objects moving across the glass surface, are the subject of future work. Specimens from each class were measured with various tools to record the size and the depth of the damage site. Measurements were also taken with the GFP and calibrated using the stress-optic coefficient determined for fused silica to obtain an image of the residual stress at the damage site. Then the specimens were fractured to measure the fracture strength. 


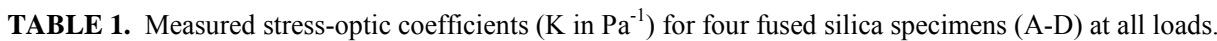

\begin{tabular}{cccccc}
\hline Load & $\mathbf{8 . 9} \mathbf{~ N}$ & $\mathbf{1 7 . 8 ~ N}$ & $\mathbf{2 6 . 7} \mathbf{~ N}$ & $\mathbf{3 5 . 6 ~ N}$ & $\mathbf{4 4 . 5 ~ N}$ \\
\hline Fused Silica A & $2.76 \mathrm{E}-12$ & $2.21 \mathrm{E}-12$ & $2.05 \mathrm{E}-12$ & $1.98 \mathrm{E}-12$ & $1.84 \mathrm{E}-12$ \\
Fused Silica B & $2.10 \mathrm{E}-12$ & $1.95 \mathrm{E}-12$ & $1.76 \mathrm{E}-12$ & $2.07 \mathrm{E}-12$ & $1.85 \mathrm{E}-12$ \\
Fused Silica C & $3.63 \mathrm{E}-12$ & $2.91 \mathrm{E}-12$ & $2.52 \mathrm{E}-12$ & $2.50 \mathrm{E}-12$ & $2.42 \mathrm{E}-12$ \\
Fused Silica D & $3.64 \mathrm{E}-12$ & $2.93 \mathrm{E}-12$ & $2.53 \mathrm{E}-12$ & $2.50 \mathrm{E}-12$ & $2.42 \mathrm{E}-12$ \\
& & & & & \\
Mean K (Pa & & & & \\
Std. Dev. & $2.43 \mathrm{E}-12$ & & & & \\
\hline
\end{tabular}

From the extensive records kept on each window, natural HVI sites that occurred during Shuttle missions were identified and cored from retired windowpanes. The core diameter choice is determined in accordance with the ASTM1499-C specification for ring-ring bi-axial strength testing.

Using the GFP 2400, photoelastic images of the optical retardation were averaged over a closed, circular path that surrounds the damage site but far enough away to be in the elastic stress field around the damage site. This procedure was designed to specifically avoid optical discontinuities at the actual damage site, and to assure that the region analyzed is in the linear stress region for the fused silica. The resulting average optical retardation is converted into average residual stress at each damage site using Equation 1. The same technique was applied to all measured damage sites, regardless of classification. The same circumference is used for each set of measurements. Figure 5 shows both (a) a visible light image and (b) optical retardation image of typical HVI site along with circular path used for averaging.

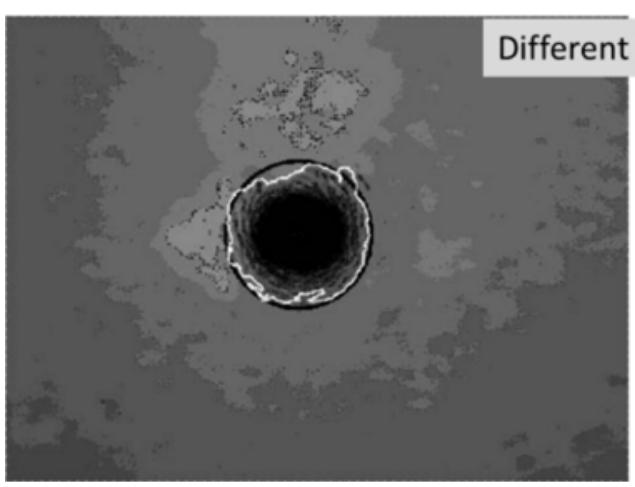

(a)

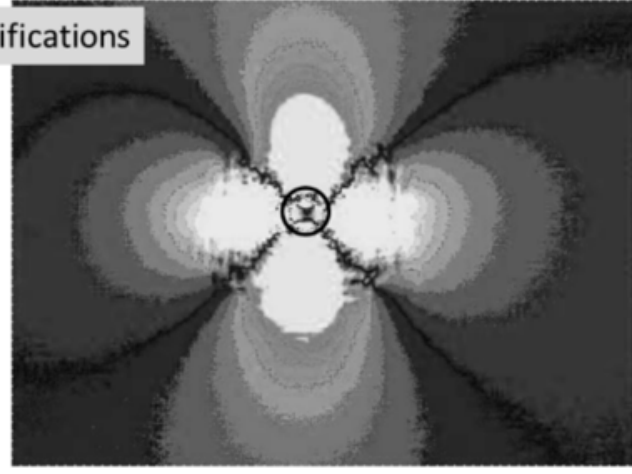

(b)

FIGURE 5. Representative images: (a) a visible light image, the white line shows the visible extent of the damage, and (b) optical retardation image of typical HVI site along with circular path (black circle) used for averaging. Note: Images do not have the same magnification.

We analyze the HVI and bruise measurement results by plotting the averaged residual stress, determined from the photoelastic stress images, versus the breakage strength. Figure 6 shows these results for five HVI specimens. A least-squares curve fit of a decaying exponential shows good correlation $\left(R^{2}=0.982\right)$ in describing the relationship between average residual strength and breakage strength. Figure 7 shows similar results for seven specimens with bruises. In the case of bruises a least-squares curve fit of a decaying exponential does not correlate as well $\left(\mathrm{R}^{2}=0.753\right)$ as in the case of the HVI damage. 


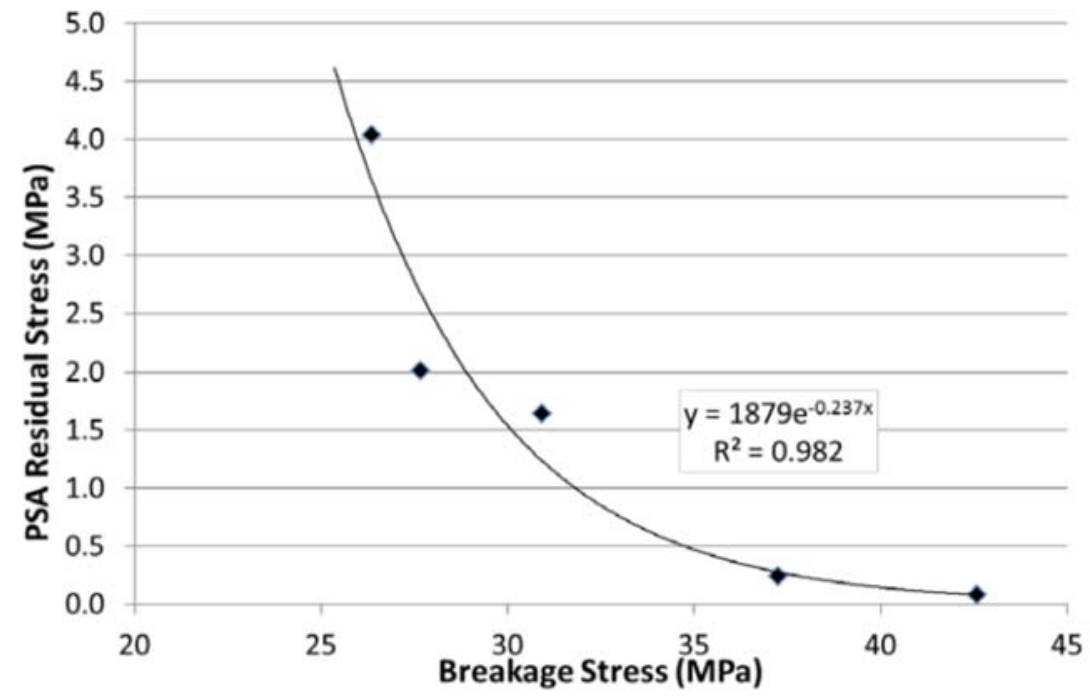

FIGURE 6. Plot of the average residual stress versus breakage strength for five HVI-type damage specimens along with a least squares curve fit of a decaying exponential.

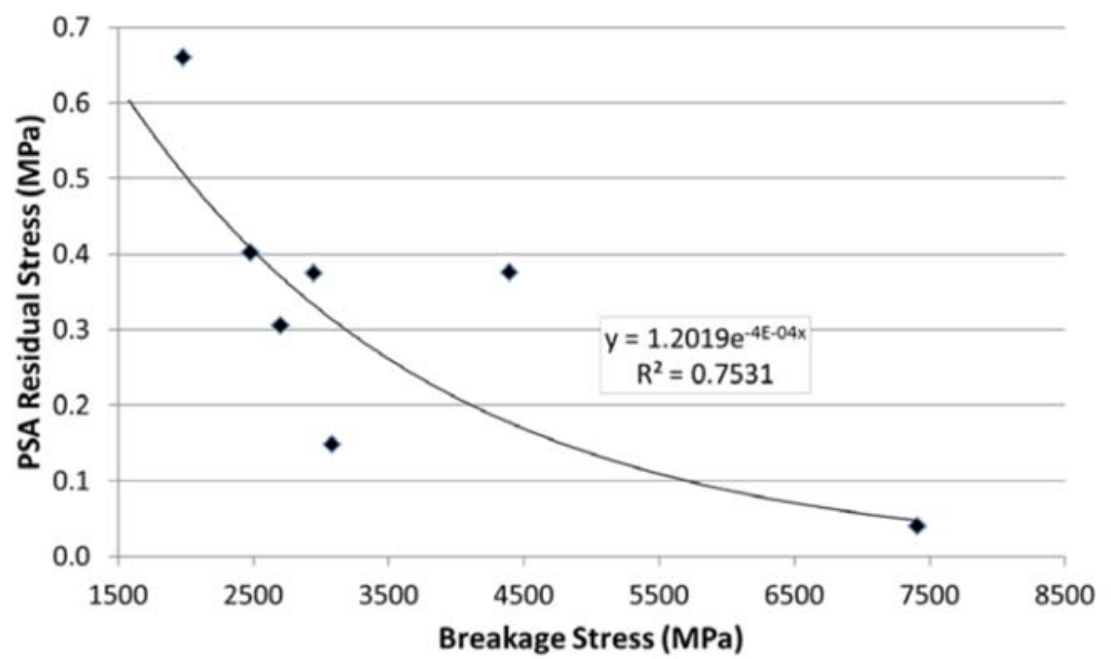

FIGURE 7. Plot of the average residual stress versus breakage strength for seven bruise-type damage specimens along with a least squares curve fit of a decaying exponential.

\section{CONCLUSIONS}

An accurate method of determining the stress-optic coefficient in transparent materials has been developed and applied to acrylic as a proof of concept case. The stress-optic coefficient for acrylic was determined within $6 \%$ of the literature value. This method was then used to determine the stress-optic coefficient for fused silica, which was determined to be $2.43 \pm 0.54 \times 10^{-12} \mathrm{~Pa}^{-1}$. Finally, using the stress-optic coefficient of fused silica, damaged fused silica specimens were examined and the residual stress after impact was determined. A line average measurement of the residual stress around the impact damage site appears to correlate well with breakage stress for HVI-type damage. But, for bruise-type damage there is much greater scatter in the data and therefore less correlation with the breakage stress. There are several potential reasons for the poorer correlation of the bruise-type data: (1) the visible 
damage region is much harder to identify, therefore at times the line average may not be uniformly in the residual stress region of the specimen, (2) because of the lower levels of damage in the case of bruise-type data, signals are near the detection limits of the system, (3) it was found that with bruise-type data damage sizes varied significantly more than with HVI-type damage, therefore using a fixed diameter circle for the line average could contribute to the larger scatter in the data. Alternative quantification approaches for the GFP data need to be investigated to fully determine its utility for predicting breakage stress.

\section{ACKNOWLEDGMENTS}

The authors would like to thank Sid Allison of NASA Langley Research center for the fabrication of the four point bending apparatus used for the determination of the stress-optic coefficient, Jon Lesniak of Stress Photonics, Inc. for the data acquisition and initial characterization of the damaged fused silica specimens, Lynda Estes of NASA-Johnson Space Center for providing the specimens, and James Lankford, Jr. of Southwest Research Institute for the glass breakage strength measurements.

\section{REFERENCES}

1. S. M. Wiederhorn and B. R. Lawn, "Strength Degradation of Glass Impacted with Sharp Particles: I, Annealed Surfaces", J. Am. Ceram. Soc., 62, pp. 66-70, (1979).

2. B. R. Lawn, D. B. Marshall, and S. M. Wiederhorn, "Strength Degradation of Glass Impacted with Sharp Particles: II, Tempered Surfaces", J. Am. Ceram. Soc., 62, pp. 71-74, (1979).

3. Edelstein, Karen S. "Orbital impacts and the Space Shuttle windshield", NASA-TM-110594, NASA. Johnson Space Center, (1995).

4. S. M. Wiederhorn, A. G. Evans, E. R. Fuller, and H. Johnson, "Application of Fracture Mechanics to Space-Shuttle Windows", J. Am. Ceram. Soc., 57, pp. 319-323, (1974).

5. B. A. Proctor, I. Whitney, and J. W. Johnson, "The strength of fused silica", Proceedings of the Royal Society of London, Series A, Mathematical and Physical Sciences, 297, pp. 534-557, (1967).

6. R. R. Burt, and E. L. Christiansen, "Hypervelocity Impact Testing of Transparent Spacecraft Materials", Intl. J. Impact Engineering, 29, pp. 153-166, (2003).

7. M. Born and E. Wolf, "Principles of Optics", 4th Edition, Pergamon Press, Oxford, UK, pp. 703-718, (1970).

8. "Standard Test Method for Monotonic Equibiaxial Flexural Strength of Advanced Ceramics at Ambient Temperatures", ASTM Standard C1499-09, ASTM International, Conshohoken, PA (2009).

9. G. Horn, J. Lesniak, T. Mackin, B. Boyce, Rev. Sci. Instrum., 76, 045108, pp. 1-10, (2005).

10. W.T. Yost, K.E. Cramer, L.R. Estes, J.A. Salem, J. Lankford, Jr., J. Lesniak, "Examination of Relationship Between Photonic Signatures and Fracture Strength of Fused Silica Used in Orbiter Windows", NASA Technical Reports Server (NTRS). NASA/TP-2011-217322 (2011).

11. J.R. Lesniak, STTR Final Report (Contract No. NAS1-97036, 2000). The initial support for its development came from Langley Research Center to Stress Photonics through the STTR Program.

12. J.R. Lesniak, M.J. Zickel, C.S. Welch, and D.F. Johnson, "An Innovative Polariscope for Photoelastic Stress Analysis", Proceedings of the Society of Experimental Mechanics Annual Conference, Bellevue, WA (June 2-4, 1997), pp. 219-224, (1997).

13. D.L. Keyes, R.R. Lamonte, D. McNally and M. Bitritto, "Polymers for Photonics", Photonics Spectra, pp. 131-134, (2011). 